\title{
A MASSIVE MOLECULAR GAS RESERVOIR IN THE $z=5.3$ SUBMILLIMETER GALAXY AzTEC-3
}

\author{
Dominik A. Riechers ${ }^{1,7}$, Peter L. Capak ${ }^{2}$, Christopher L. Carilli ${ }^{3}$, Pierre Cox ${ }^{4}$, Roberto Neri ${ }^{4}$, Nicholas Z. Scoville ${ }^{1}$, \\ EVA SCHINNERER ${ }^{5}$, FRANK BERTOLDI ${ }^{6}$, AND LIN YAN ${ }^{2}$ \\ ${ }^{1}$ Astronomy Department, California Institute of Technology, MC 249-17, 1200 East California Boulevard, Pasadena, CA 91125, USA; dr@ caltech.edu \\ ${ }^{2}$ Spitzer Science Center, California Institute of Technology, MC 220-6, 1200 East California Boulevard, Pasadena, CA 91125, USA \\ ${ }^{3}$ National Radio Astronomy Observatory, P.O. Box O, Socorro, NM 87801, USA \\ ${ }^{4}$ Institut de RadioAstronomie Millimétrique, 300 Rue de la Piscine, Domaine Universitaire, 38406 Saint Martin d'Héres, France \\ ${ }_{5}^{5}$ Max-Planck-Institut für Astronomie, Königstuhl 17, D-69117 Heidelberg, Germany \\ ${ }^{6}$ Argelander-Institut für Astronomie, Universität Bonn, Auf dem Hügel 71, Bonn, D-53121, Germany \\ Received 2010 June 28; accepted 2010 July 30; published 2010 August 16
}

\begin{abstract}
We report the detection of $\mathrm{CO} J=2 \rightarrow 1,5 \rightarrow 4$, and $6 \rightarrow 5$ emission in the highest-redshift submillimeter galaxy (SMG) AzTEC-3 at $z=5.298$, using the Expanded Very Large Array and the Plateau de Bure Interferometer. These observations ultimately confirm the redshift, making AzTEC-3 the most submillimeter-luminous galaxy in a massive $z \simeq 5.3$ protocluster structure in the COSMOS field. The strength of the CO line emission reveals a large molecular gas reservoir with a mass of $5.3 \times 10^{10}\left(\alpha_{\mathrm{CO}} / 0.8\right) M_{\odot}$, which can maintain the intense $1800 M_{\odot} \mathrm{yr}^{-1}$ starburst in this system for at least $30 \mathrm{Myr}$, increasing the stellar mass by up to a factor of six in the process. This gas mass is comparable to "typical" $z \sim 2$ SMGs and constitutes $\gtrsim 80 \%$ of the baryonic mass (gas+stars) and $30 \%-80 \%$ of the total (dynamical) mass in this galaxy. The molecular gas reservoir has a radius of $<4 \mathrm{kpc}$ and likely consists of a "diffuse", low-excitation component, containing (at least) $1 / 3$ of the gas mass (depending on the relative conversion factor $\alpha_{\mathrm{CO}}$ ), and a "dense", high-excitation component, containing $\sim 2 / 3$ of the mass. The likely presence of a substantial diffuse component besides highly excited gas suggests different properties between the star-forming environments in $z>4$ SMGs and $z>4$ quasar host galaxies, which perhaps trace different evolutionary stages. The discovery of a massive, metal-enriched gas reservoir in an SMG at the heart of a large $z=5.3$ protocluster considerably enhances our understanding of early massive galaxy formation, pushing back to a cosmic epoch where the universe was less than $1 / 12$ of its present age.
\end{abstract}

Key words: cosmology: observations - galaxies: active - galaxies: formation - galaxies: high-redshift - galaxies: starburst - radio lines: galaxies

Online-only material: color figures

\section{INTRODUCTION}

Our understanding of the physical properties of submillimeter galaxies (SMGs; see review by Blain et al. 2002) is of key importance to studies of the early formation and evolution of massive galaxies, as they are the likely progenitors of the most massive galaxies in the present-day universe. SMGs typically represent compact, intense ( $>1000 M_{\odot} \mathrm{yr}^{-1}$ ), rather short-lived $(<100 \mathrm{Myr})$ starbursts with rapid gas consumption through high star formation efficiencies that are commonly associated with ongoing major mergers. Their star formation rates (SFRs) exceed those of "normal" high- $z$ galaxies with comparable stellar mass $\left(M_{\star}\right)$ by more than an order of magnitude at $z \sim 2$ (e.g., Daddi et al. 2009), making them a comparatively rare, but cosmologically important population. The extreme star formation events in SMGs are typically associated with large amounts of gas and dust which often obscure the stellar light and star formation at rest-frame ultraviolet (UV) to optical wavelengths, making their identification at such wavelengths notoriously difficult.

The most insightful way to study SMGs and their star formation properties thus usually is through the dust-reprocessed UV light from newly formed stars that is re-emitted in the farinfrared (FIR) continuum (a measure of the SFR) and through line emission from molecular gas (typically $\mathrm{CO}$ ), the fuel for star formation. Molecular gas was detected in $>20$ SMGs to date, revealing large gas reservoirs of $>10^{10} M_{\odot}$ in most cases (see Solomon \& Vanden Bout 2005 for a review).

Recently, Capak et al. (2010) discovered AzTEC-3, an SMG at an unprecedented redshift of $z=5.3$. AzTEC-3 is not only the most distant SMG known to date, but also resides in a massive, overdense, protocluster environment extending out to $>2 \mathrm{Mpc}$, with two companions within $12.2 \mathrm{kpc}$. AzTEC-3 hosts an $1800 M_{\odot} \mathrm{yr}^{-1}$ starburst ${ }^{8}$ exhibiting an FIR luminosity of $L_{\mathrm{FIR}}=(1.7 \pm 0.8) \times 10^{13} L_{\odot}$ that likely produced a substantial fraction of its current stellar mass of $M_{\star}=(1.0 \pm 0.2) \times$ $10^{10} M_{\odot}$. The unusual nature of AzTEC-3 and its potential major implications for massive galaxy formation at very early cosmic epochs have initiated an in-depth study of its physical properties and cosmic environment.

In this Letter, we report the detection of $\mathrm{CO}(J=2 \rightarrow 1), \mathrm{CO}(J$ $=5 \rightarrow 4)$, and $\mathrm{CO}(J=6 \rightarrow 5)$ emission toward AzTEC-3 $(z=$ 5.298), using the Expanded Very Large Array (EVLA) and the Plateau de Bure Interferometer (PdBI). We use a concordance, flat $\Lambda \mathrm{CDM}$ cosmology throughout, with $H_{0}=71 \mathrm{~km} \mathrm{~s}^{-1} \mathrm{Mpc}^{-1}$, $\Omega_{\mathrm{M}}=0.27$, and $\Omega_{\Lambda}=0.73$ (Spergel et al. 2003, 2007).

\section{OBSERVATIONS}

\subsection{Plateau de Bure Interferometer}

We observed the $\mathrm{CO}(J=5 \rightarrow 4)\left(v_{\text {rest }}=576.2679305 \mathrm{GHz}\right)$ and $\mathrm{CO}(J=6 \rightarrow 5)\left(v_{\text {rest }}=691.4730763 \mathrm{GHz}\right)$ emission

\footnotetext{
7 Hubble Fellow.
}

\footnotetext{
8 Assuming a Chabrier (2003) stellar initial mass function (IMF).
} 


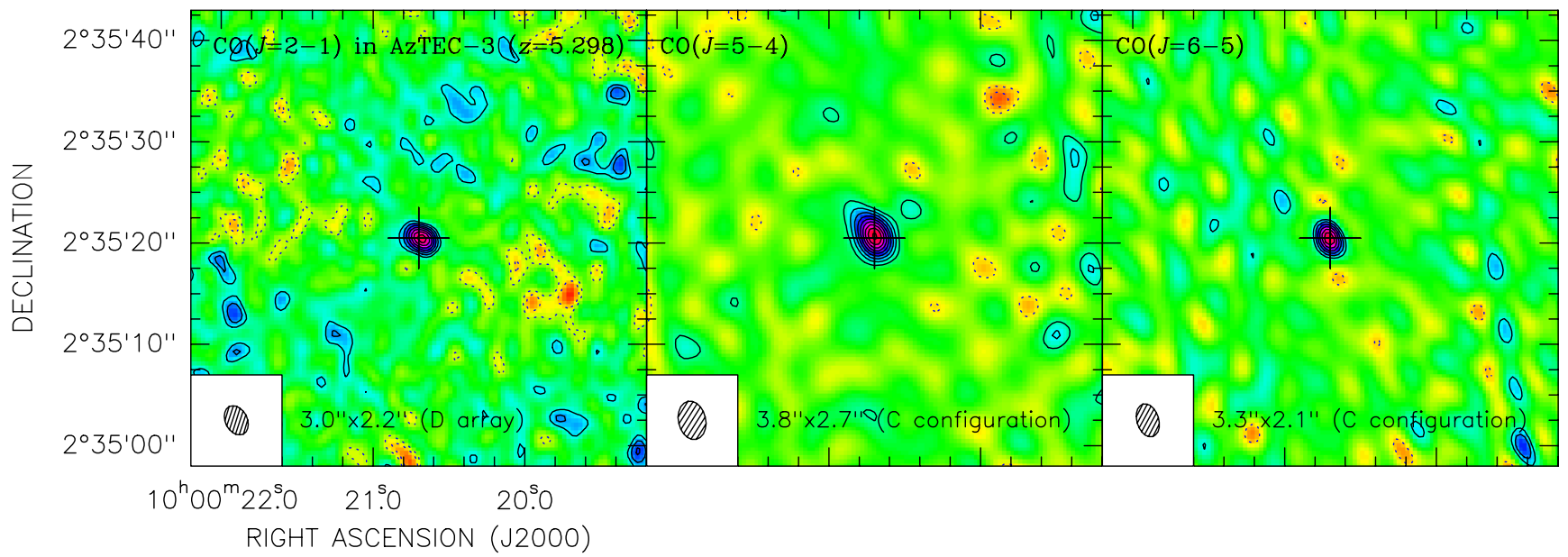

Figure 1. Velocity-integrated EVLA/PdBI maps of the $\mathrm{CO}(J=2 \rightarrow 1)$ (left), $\mathrm{CO}(J=5 \rightarrow 4)$ (middle), and $\mathrm{CO}(J=6 \rightarrow 5)$ (right) line emission over $950 / 852 / 874 \mathrm{~km} \mathrm{~s}{ }^{-1}$ toward AzTEC-3. At resolutions of 3'. $0 \times 22^{\prime \prime} .2,3$, $8 \times 22^{\prime \prime} .7$, and 3'.3 $\times 22^{\prime \prime} .1$ (as indicated in the bottom left corner of each panel), the emission remains unresolved. The cross indicates the position of the submillimeter continuum emission (Younger et al. 2007). Contours are shown in steps of $1 \sigma=0.045 / 0.10 / 0.22 \mathrm{mJy}$ beam ${ }^{-1}$, starting at $\pm 2 \sigma$.

(A color version of this figure is available in the online journal.)

lines toward AzTEC-3 using the PdBI. At $z=5.298$, these lines are redshifted to 91.5001 and $109.7925 \mathrm{GHz}$ (3.3 and $2.7 \mathrm{~mm}$ ). Observations were carried out under good $3 \mathrm{~mm}$ weather conditions in the $6 \mathrm{C}$ configuration on 2010 April 1 and 2, resulting in 4.4 and $3.1 \mathrm{hr}$ on-source time for the $\mathrm{CO}$ $J=5 \rightarrow 4$ and $6 \rightarrow 5$ lines, respectively. The nearby quasar B0906+015 (distance to AzTEC-3: 12:8) was observed every 22.5 minutes for pointing, secondary amplitude and phase calibration. For primary flux calibration, the standard calibrators MWC349 and 3C84 were observed, leading to a calibration that is accurate within $\lesssim 10 \%$. Observations were set up using a total bandwidth of $1 \mathrm{GHz}$ (dual polarization; corresponding to $\sim 3300 / 2700 \mathrm{~km} \mathrm{~s}^{-1}$ at $3.3 / 2.7 \mathrm{~mm}$ ) with the current correlator and a total bandwidth of $3.6 \mathrm{GHz}$ (dual polarization) with the new WideX correlator (recorded simultaneously).

For data reduction and analysis, the GILDAS package was used. All data were mapped using "natural" weighting. The CO $J=5 \rightarrow 4 / 6 \rightarrow 5$ data result in a final rms of $0.52 /$ $1.27 \mathrm{mJy}$ beam $^{-1}$ per $33 / 27 \mathrm{~km} \mathrm{~s}^{-1}(10 \mathrm{MHz})$ channel and $0.05 / 0.13$ mJy beam $^{-1}\left(0.028 / 0.066 \mathrm{mJy} \mathrm{beam}^{-1}\right)$ over the entire $1 \mathrm{GHz}(3.6 \mathrm{GHz})$ bandwidth. Maps of the velocity-integrated $\mathrm{CO} J=5 \rightarrow 4 / 6 \rightarrow 5$ line emission yield synthesized clean beam sizes of 3." $8 \times 2$.". 7 and 3.'3 $\times 2$ 2. 1 and rms noise values of $0.10 / 0.22 \mathrm{mJy}$ beam $^{-1}$ over $852 / 874 \mathrm{~km} \mathrm{~s}^{-1}(260 / 320 \mathrm{MHz})$.

\subsection{Expanded Very Large Array}

We observed the $\mathrm{CO}(J=2 \rightarrow 1)\left(v_{\text {rest }}=230.53799 \mathrm{GHz}\right)$ emission line toward AzTEC-3 using the EVLA. At $z=5.298$, this line is redshifted to $36.6049 \mathrm{GHz}(8.2 \mathrm{~mm})$. Observations were carried out under good $9 \mathrm{~mm}$ weather conditions in D array on 2010 May 24 and 30, resulting in $6.3 \mathrm{hr}$ on-source time with 16 antennas (equivalent to $2.2 \mathrm{hr}$ with 27 antennas) after rejection of bad data. The nearby quasar J1018+0530 (distance to AzTEC-3: 5.4) was observed every 7 minutes for pointing, secondary amplitude and phase calibration. For primary flux calibration, the standard calibrator 3C286 was observed, leading to a calibration that is accurate within $\lesssim 10 \%$. Observations were set up using a total bandwidth of $256 \mathrm{MHz}$ (dual polarization; corresponding to $\sim 2100 \mathrm{~km} \mathrm{~s}^{-1}$ at $8.2 \mathrm{~mm}$ ) with the WIDAR correlator.

For data reduction and analysis, the AIPS package was used. All data were mapped using "natural" weighting. The data result in a final rms of $0.20 \mathrm{mJy}^{-1}$ beam $^{-1}$ per $49 \mathrm{~km} \mathrm{~s}^{-1}(6 \mathrm{MHz})$ channel and $0.030 \mathrm{mJy}^{-1}$ beam $^{-1}$ over the entire $256 \mathrm{MHz}$ bandwidth. Maps of the velocity-integrated CO $J=2 \rightarrow 1$ line emission yield a synthesized clean beam size of 3 .' $0 \times 2$ '. 2 at an rms noise level of $0.045 \mathrm{mJy}_{\text {beam }}{ }^{-1}$ over $950 \mathrm{~km} \mathrm{~s}^{-1}(116 \mathrm{MHz})$.

\section{RESULTS}

We have detected $\mathrm{CO}(J=2 \rightarrow 1), \mathrm{CO}(J=5 \rightarrow 4)$, and $\mathrm{CO}(J=6 \rightarrow 5)$ line emission toward the $z=5.298$ SMG AzTEC3 at $8 \sigma, 10 \sigma$, and $7 \sigma$ significance (Figure 1). The $\mathrm{CO} J=$ $5 \rightarrow 4$ and $6 \rightarrow 5$ data yield a combined significance of $12 \sigma$. We do not detect the underlying continuum emission down to $2 \sigma$ limits of $0.13 / 0.31 \mathrm{mJy}^{-13}$ beam $^{-1}$ (WideX correlator: 0.08 / $0.14 \mathrm{mJy}$ beam $^{-1}$ ) at $3.3 / 2.7 \mathrm{~mm}$ (rest-frame $520 / 434 \mu \mathrm{m}$ ). A $2 \sigma-3 \sigma$ peak is present in the $8.2 \mathrm{~mm}$ map (rest-frame $1.3 \mathrm{~mm}$ ), but offset from the $\mathrm{CO}$ peak position by $\sim 1^{\prime \prime}$. We thus consider the underlying continuum at this wavelength undetected down to $\sim 0.09$ mJy beam $^{-1}$.

From Gaussian fitting to the line profiles (Figure 2), we obtain $\mathrm{CO} J=2 \rightarrow 1,5 \rightarrow 4$, and $6 \rightarrow 5$ line peak strengths of $0.45 \pm$ $0.07,{ }^{9} 1.78 \pm 0.17$, and $2.64 \pm 0.36 \mathrm{mJy}$ at an FWHM of $487 \pm 58 \mathrm{~km} \mathrm{~s}^{-1}$, centered at a (weighted median) redshift of $z=5.2979 \pm 0.0004$ (consistent with the optical redshift; Capak et al. 2010). This corresponds to velocity-integrated emission line strengths of $0.23 \pm 0.03,0.92 \pm 0.09$, and $1.36 \pm$ $0.19 \mathrm{Jy} \mathrm{km} \mathrm{s}{ }^{-1}$, i.e., line luminosities of $L_{\mathrm{CO}(2-1)}^{\prime}=(5.84 \pm$ $0.78), L_{\mathrm{CO}(5-4)}^{\prime}=(3.70 \pm 0.37)$, and $L_{\mathrm{CO}(6-5)}^{\prime}=(3.82 \pm 0.54) \times$ $10^{10} \mathrm{~K} \mathrm{~km} \mathrm{~s}^{-1} \mathrm{pc}^{2}$. This also corresponds to $\mathrm{CO} J=6 \rightarrow 5 / 5 \rightarrow 4$ and $\mathrm{CO} J=5 \rightarrow 4 / 2 \rightarrow 1$ line brightness temperature ratios of $r_{65}=1.03 \pm 0.16$ (i.e., consistent with thermalized) and $r_{52}=$ $0.63 \pm 0.10$.

\section{ANALYSIS AND DISCUSSION}

\subsection{Origin of the $\mathrm{CO}$ and Submillimeter Emission}

Within the size of the $\mathrm{CO}$ beam, there are three galaxies with colors that are consistent with the CO redshift of AzTEC-3. By

\footnotetext{
9 The fit also suggests an underlying continuum component of $0.09 \pm$ $0.04 \mathrm{mJy}$, consistent with the marginal peak seen in the map.
} 


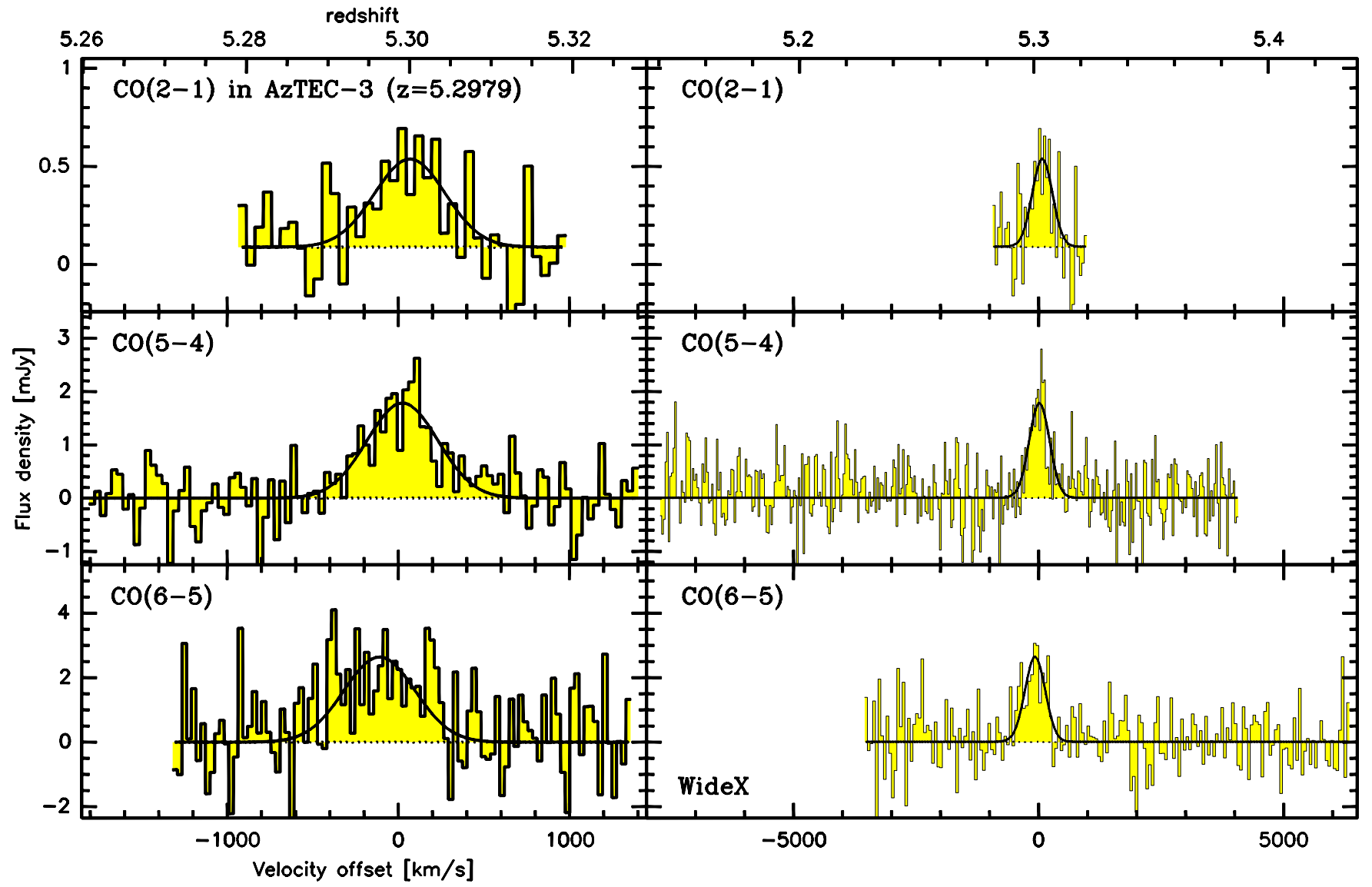

Figure 2. Left: EVLA/PdBI $\mathrm{CO}(J=2 \rightarrow 1)$ (top), $\mathrm{CO}(J=5 \rightarrow 4)$ (middle), and $\mathrm{CO}(J=6 \rightarrow 5)$ (bottom) spectra of AzTEC-3 at $6 / 10 / 10 \mathrm{MHz}\left(49 / 33 / 27 \mathrm{~km} \mathrm{~s}{ }^{-1}\right)$ resolution (histograms), along with Gaussian fits to the line emission (black curves). The velocity scale is relative to the source's redshift of $z=5.2979 \pm 0.0004$, as measured from the molecular line emission. Right: same, but showing the PdBI data recorded with the WideX correlator (CO J=6 $\rightarrow 5$ is re-binned to $20 \mathrm{MHz}$ ).

(A color version of this figure is available in the online journal.)

combining the integrated emission of the $\mathrm{CO} J=5 \rightarrow 4$ and $6 \rightarrow 5$ lines detected at $12 \sigma$ significance and fitting the $u-v$ data with a circular Gaussian, we find that the source is unresolved

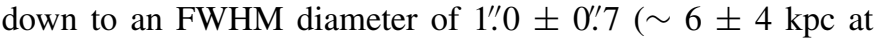
$z=5.3)$. This is consistent with a limit of $1^{\prime \prime} 3_{-1^{\prime \prime}, 3}^{+0^{\prime \prime}}\left(\sim 8_{-8}^{+5} \mathrm{kpc}\right)$ as derived from the $\mathrm{CO} J=2 \rightarrow 1$ data. Only one of the three color-selected galaxies falls within this smaller area, identifying the $i \sim 26$ galaxy targeted by the optical spectroscopy as the real counterpart of the $\mathrm{CO}$ emission (Figure 3 ). The astrometric accuracy of the $\mathrm{CO}(J=2 \rightarrow 1)$ and combined $\mathrm{CO} J=5 \rightarrow 4$ and $6 \rightarrow 5$ detections is $0{ }^{\prime} \cdot 15$ and $0^{\prime \prime} 12$. The peak position of the CO emission is consistent with that of the $890 \mu \mathrm{m}$ continuum emission (astrometric accuracy: 0"' 14; Younger et al. 2007; see also Scott et al. 2008) and the $3.6 \mu \mathrm{m}$ emission (astrometric accuracy: 0.'2; Sanders et al. 2007) within the relative uncertainties (Figure 3). Interestingly, the emission at all these wavelengths is offset from the HST/ACS $i$-band image of the source by $\sim 0^{\prime \prime} .5(3 \mathrm{kpc})$, but is consistent with a marginal, much fainter peak in the rest-frame UV image (which we interpret to be part of the same galaxy; inset in Figure 3). Given the 0 '. 1 relative astrometric accuracy of the Hubble Space Telescope (HST) data, this offset is formally significant. We conclude that this offset is due to a combination of lacking surface brightness sensitivity and dust obscuration in the rest-frame UV image, a similar effect as seen in the $z=4.055$ SMG GN20 (e.g., Carilli et al. 2010). This effect also explains the substantial difference between the UV- and farIR-derived SFRs of AzTEC-3 (Capak et al. 2010), suggesting that the regions of most intense star formation are highly dust obscured.

\subsection{CO Line Excitation}

Based on the CO excitation ladder of AzTEC-3, we can constrain the line radiative transfer through large velocity gradient (LVG) models, treating the gas kinetic temperature and density as free parameters. For all calculations, the $\mathrm{H}_{2}$ ortho-to-para ratio was fixed to $3: 1$, the cosmic microwave background temperature was fixed to $17.16 \mathrm{~K}$ (at $z=5.298$ ), and the Flower (2001) CO collision rates were used. We adopted a $\mathrm{CO}$ abundance per velocity gradient of $[\mathrm{CO}] /(d v / d r)=$ $1 \times 10^{-5} \mathrm{pc}\left(\mathrm{km} / \mathrm{s}^{-1}\right)^{-1}$ (e.g., Weiß et al. 2005, 2007; Riechers et al. 2006).

The data are poorly fit by single-component models, which underpredict the $\operatorname{CO}(J=2 \rightarrow 1)$ flux by $\sim 30 \%$. The data can be fitted reasonably well with two gas components, which are represented by a "diffuse", low-excitation component with a kinetic temperature of $T_{\mathrm{kin}}=30 \mathrm{~K}$ and a gas density of $\rho_{\text {gas }}=10^{2.5} \mathrm{~cm}^{-3}$, and a more "dense", high-excitation component with $T_{\text {kin }}=45 \mathrm{~K}$ and $\rho_{\text {gas }}=10^{4.5} \mathrm{~cm}^{-3}$ in our model (Figure 4). Assuming that the molecular gas is distributed in a face-on, circular disk, the observed limits of $1^{\prime \prime} .3$ and $1^{\prime \prime} .0$ on the sizes of these low- and high-excitation gas components yield CO disk filling factors of $\gtrsim 75 \%$ and $\gtrsim 10 \%$. The "diffuse" gas component has physical properties comparable to those of nearby spiral galaxies and "normal" high- $z$ star-forming galaxies (e.g., Dannerbauer et al. 2009) and contributes $\sim 35 \%$ to 


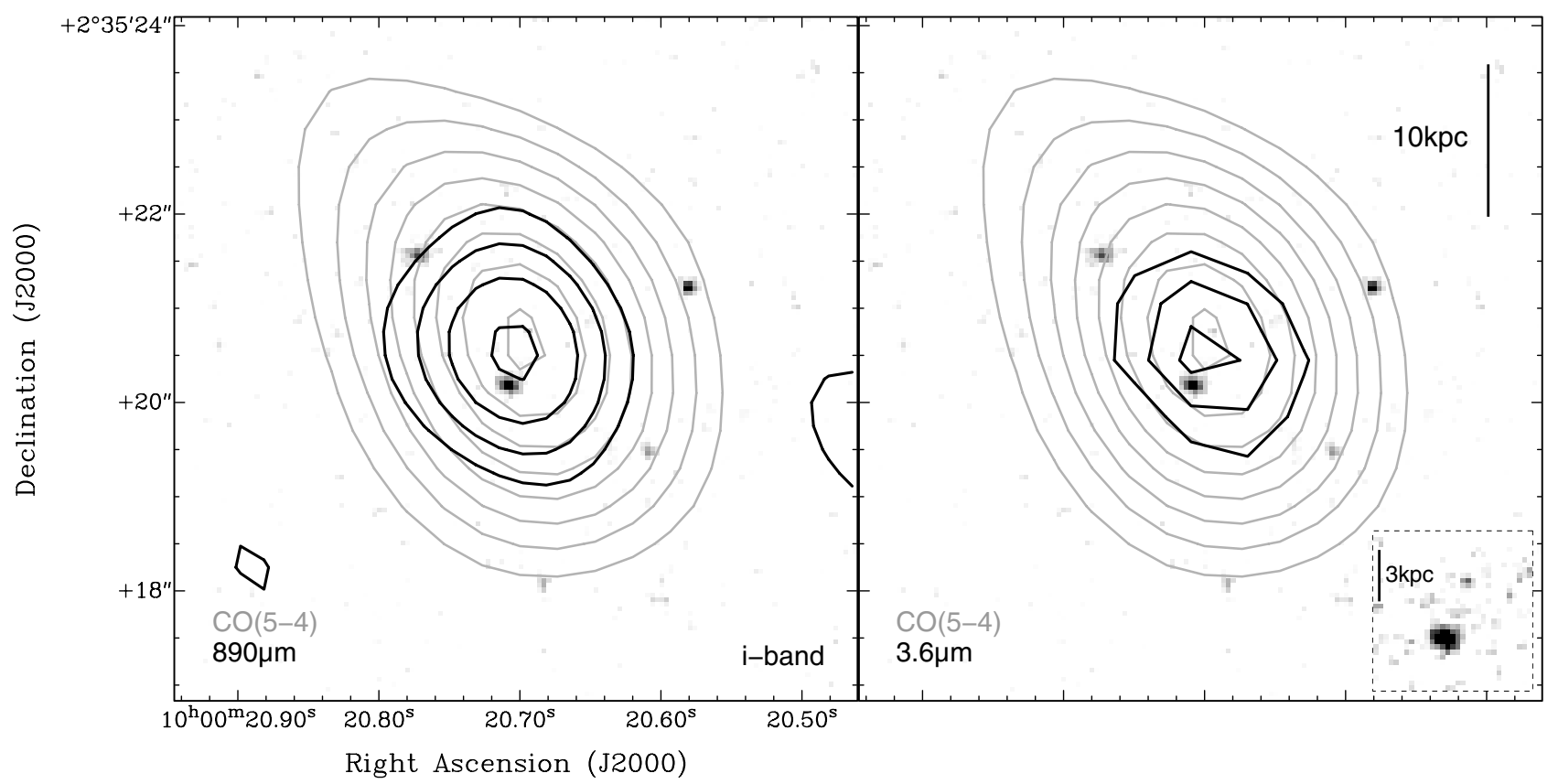

Figure 3. Overlays of the $\mathrm{CO}(J=5 \rightarrow 4)$ emission (gray contours) and SMA $890 \mu \mathrm{m}$ (left; 2.'7 $\times 2$ 2.' 1 resolution; Younger et al. 2007), as well as Spitzer/IRAC $3.6 \mu \mathrm{m}$ (right; PSF FWHM = 1". 6 ; from Sanders et al. 2007) emission (black contours in each panel), with an HST/ACS $i$-band image (gray scale and inset; from Scoville et al. 2007).

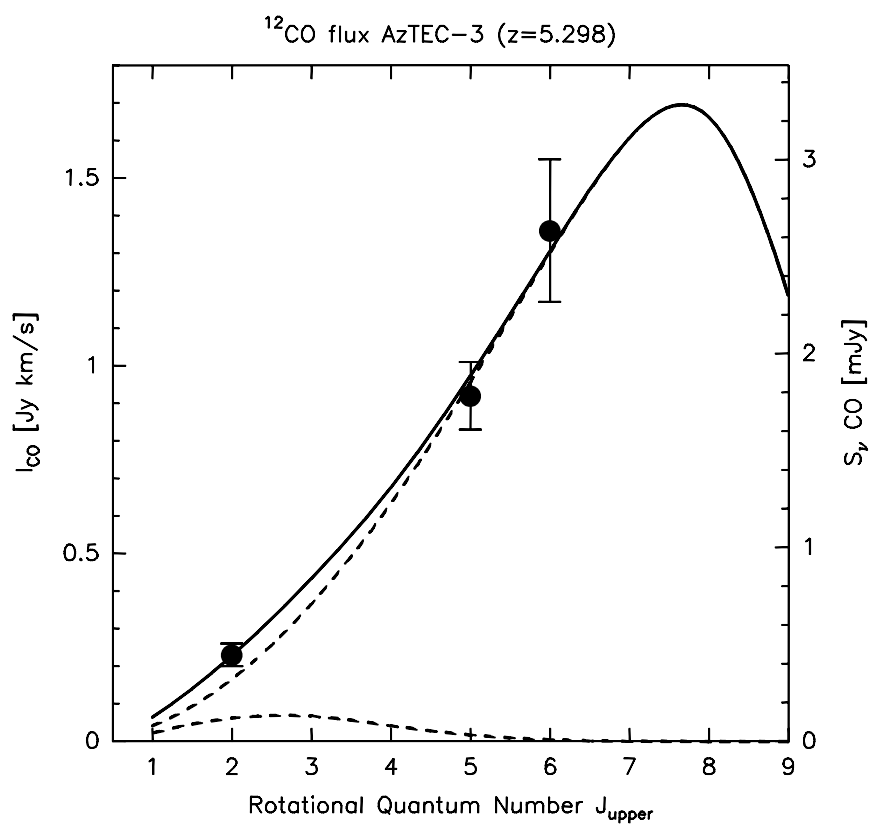

Figure 4. CO excitation ladder (points) and LVG model (lines) for AzTEC-3. The model (solid line) consists of two gas components (dashed lines): a Milky Way like, low-excitation component with a kinetic temperature of $T_{\text {kin }}=30 \mathrm{~K}$ and a gas density of $\rho_{\text {gas }}=10^{2.5} \mathrm{~cm}^{-3}$, and a ULIRG-like, high-excitation component with $T_{\text {kin }}=45 \mathrm{~K}$ and $\rho_{\text {gas }}=10^{4.5} \mathrm{~cm}^{-3}$.

the model-predicted $\mathrm{CO}(J=1 \rightarrow 0)$ flux. The "dense" gas component has properties comparable to ultra-luminous infrared galaxy (ULIRG) nuclei and high- $z$ FIR-luminous quasars (e.g., Riechers et al. 2006, 2009), contributing $\sim 65 \%$ to the $\mathrm{CO}(J=$ $1 \rightarrow 0$ ) flux.

The model suggests an excitation-corrected $\mathrm{CO}(J=1 \rightarrow 0)$ line luminosity of $L_{\mathrm{CO}}^{\prime}=6.6 \times 10^{10} \mathrm{~K} \mathrm{~km} \mathrm{~s}^{-1} \mathrm{pc}^{2}$ and $L_{\mathrm{FIR}} / L_{\mathrm{CO}}^{\prime}$ $=260 L_{\odot}\left(\mathrm{K} \mathrm{km} \mathrm{s}^{-1} \mathrm{pc}^{2}\right)^{-1}$. This luminosity ratio is comparable to "typical" $z>2$ SMGs and quasar host galaxies (e.g., Greve et al. 2005; Riechers et al. 2006).

\subsection{Total Molecular Gas Mass and Dynamical Mass}

Given the gas excitation conditions in AzTEC-3, we derive the total molecular gas mass based on a ULIRG conversion factor of $\alpha_{\mathrm{CO}}=0.8 M_{\odot}\left(\mathrm{K} \mathrm{km} \mathrm{s}^{-1} \mathrm{pc}^{2}\right)^{-1}$ from $L_{\mathrm{CO}}^{\prime}$ to $M_{\mathrm{gas}}$ (Downes \& Solomon 1998), yielding $M_{\text {gas }}=5.3 \times 10^{10} M_{\odot} \cdot{ }^{10}$ This corresponds to $5.3 \times M_{\star}$ in this system, implying that the baryonic mass is dominated by the gaseous component. This means that the intense, $1800 M_{\odot} \mathrm{yr}^{-1}$ starburst in this galaxy has sufficient fuel to more than sextuple $M_{\star}$ throughout the remainder of its duration. This also sets the gas depletion timescale $\tau_{\mathrm{dep}}=M_{\mathrm{gas}} / \mathrm{SFR}$ to $30 \mathrm{Myr}$, comparable to lower redshift SMGs (e.g., Greve et al. 2005; Schinnerer et al. 2008) and $z>4$ quasar host galaxies (e.g., Riechers et al. 2008).

Adopting the size limit of $1^{\prime \prime} 0(\sim 6.2 \mathrm{kpc})$, this also corresponds to a limiting average gas surface density of $\Sigma_{\text {gas }} \gtrsim 1.7$ $\times 10^{9} M_{\odot} \mathrm{kpc}^{-2}$. This is comparable to the values found for $z \gtrsim 2$ SMGs (typically $2 \times 10^{9} M_{\odot} \mathrm{kpc}^{-2}$; Tacconi et al. $2006,2008)$ and about half of the peak gas density of the $z=6.42$ quasar J1148+5251 $\left(3.5 \times 10^{9} M_{\odot} \mathrm{kpc}^{-2}\right.$; Riechers et al. 2009). Assuming an intrinsic $\Sigma_{\text {gas }}$ in this range (i.e., $\sim 2.7 \times$ $10^{9} \quad M_{\odot} \mathrm{kpc}^{-2}$ ), this may indicate that the gas reservoir in AzTEC-3 has an intrinsic circular gas disk-equivalent radius of $r_{0} \sim 2.5 \mathrm{kpc}$.

Based on the velocity and size information extracted from the $\mathrm{CO}$ data, we can determine a value for the dynamical mass $\left(M_{\mathrm{dyn}}\right)$ of AzTEC-3. Given that the contributions from the central supermassive black hole $\left(M_{\mathrm{BH}} \lesssim 0.1 \% M_{\star} \propto 10^{7} M_{\odot}\right.$; Alexander et al. 2008) and dust $\left(M_{\text {dust }} \lesssim 1 \%-2 \% M_{\text {gas }} ;\right.$ e.g., Michalowski et al. 2010) to the total mass budget are expected to be small, we here approximate $M_{\mathrm{tot}} \simeq M_{\mathrm{dyn}} \simeq M_{\mathrm{gas}}+M_{\star}+M_{\mathrm{DM}}$, where $M_{\mathrm{DM}}$ is the contribution from dark matter. We further define the gas

\footnotetext{
${ }^{10}$ Assuming a Milky Way like $\alpha_{\mathrm{CO}}=3.5 M_{\odot}\left(\mathrm{K} \mathrm{km} \mathrm{s}^{-1} \mathrm{pc}^{2}\right)^{-1}$ for the
} low-excitation component would increase $M_{\text {gas }}$ by a factor of 2.2 . 


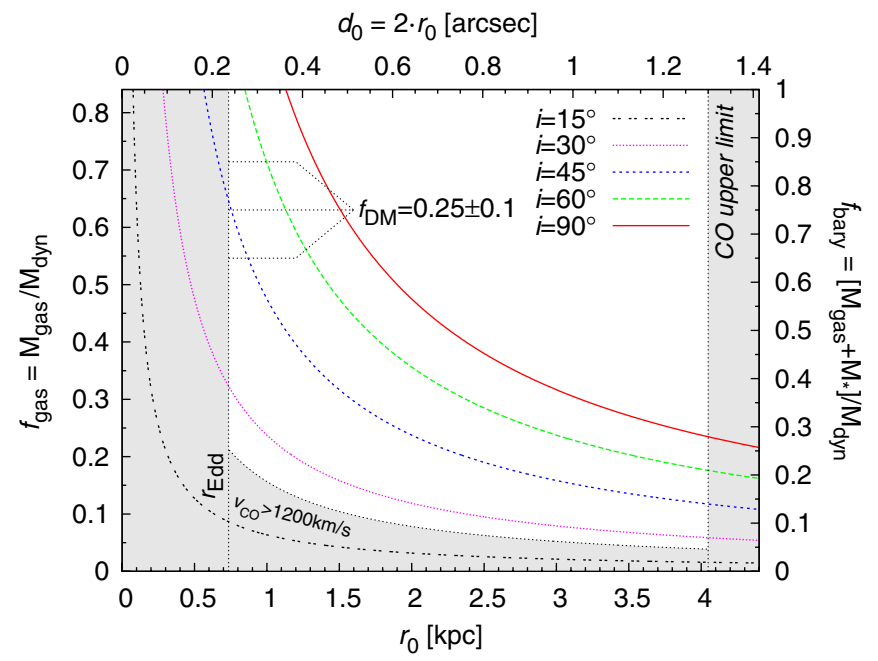

Figure 5. Constraints on the gas $\left(f_{\text {gas }}\right)$ and baryonic $\left(f_{\text {bary }}\right)$ mass fractions for different line-of-sight inclination angles $i$ for AzTEC-3 (where $i=90^{\circ}$ corresponds to edge-on). $r_{0}$ is the equivalent radius of a circular disk used for deriving $M_{\mathrm{dyn}}$. The upper limit derived from the $\mathrm{CO}$ data and the lower limit derived from the Eddington limit on $L_{\mathrm{FIR}}\left(r_{\mathrm{Edd}}\right)$ are indicated by the vertical lines and shaded areas. A lower limit on $i$ is determined by assuming that $v_{\mathrm{CO}}=\mathrm{d} v_{\mathrm{FWHM}} \sin ^{-1} i \leqslant 1200 \mathrm{~km} \mathrm{~s}^{-1}$, as indicated by the lower dotted curve and shaded area. The allowed range of $i$ for an assumed dark matter fraction of $f_{\mathrm{DM}}=1-f_{\text {bary }}=25 \% \pm 10 \%$ (corresponding to $f_{\text {gas }}=63 \% \pm 8 \%$ ) is indicated by the thin dotted horizontal/arrow-shaped lines.

(A color version of this figure is available in the online journal.)

mass fraction as $f_{\text {gas }}=M_{\text {gas }} / M_{\text {dyn }}$ and the baryonic mass fraction as $f_{\text {bary }}=1-f_{\mathrm{DM}}=\left[M_{\mathrm{gas}}+M_{\star}\right] / M_{\text {dyn }}$.

In Figure $5, f_{\text {gas }}$ and $f_{\text {bary }}$ are shown as a function of $r_{0}$ and the inclination $i$ of the gas disk. For reference, $r_{0}=2.5 \mathrm{kpc}$ corresponds to $M_{\text {dyn }} \sin ^{2} i=1.4 \times 10^{11} M_{\odot}$, i.e., $f_{\text {gas }} \sim 0.4$ and $f_{\text {bary }} \sim 0.45$. $^{11}$ From the observations of the gas and dust in this galaxy, we can infer three main constraints on $M_{\text {dyn }}$ (shaded regions in Figure 5). First, the $\mathrm{CO}$ observations place an upper limit of 1 .'3 on $r_{0}$. Second, of the $>20$ SMGs detected in CO emission to date, none have an FWHM linewidth of $>1200 \mathrm{~km} \mathrm{~s}^{-1}$ (median: $530 \mathrm{~km} \mathrm{~s}^{-1}$; Coppin et al. 2008). Taking this as an upper limit on the intrinsic, inclinationcorrected linewidth $v_{\mathrm{CO}}=\mathrm{d} v_{\text {FWHM }} \sin ^{-1} i$ yields a lower limit of $i>24^{\circ}$ ( $i=0^{\circ}$ corresponds to face-on). Third, assuming that the starburst disk is supported by radiation pressure on dust grains yields an Eddington limit on the FIR flux of $F_{\mathrm{FIR}} \simeq 10^{13} L_{\odot} \mathrm{kpc}^{-2}$ (e.g., Scoville 2003; Thompson et al. $2005)$. Assuming that all $L_{\text {FIR }}$ is due to star formation in the gasrich reservoir, this yields a lower limit on $r_{0}$ of $r_{\text {Edd }}=735 \mathrm{pc}$. Note that the corresponding dust brightness temperature limit of $T_{\text {dust }}^{\mathrm{Edd}}=88 \mathrm{~K}$ is more than twice as high as the $T_{\text {dust }}$ obtained from fitting the spectral energy distribution (Capak et al. 2010), which may indicate that $F_{\text {FIR }}$ in AzTEC-3 is well below the Eddington limit on average, contrary to what is seen, e.g., in the center of the $z=6.42$ quasar J1148+5251 (Walter et al. 2009; Riechers et al. 2009).

Based on these constraints on $M_{\mathrm{dyn}}$, and assuming that $\alpha_{\mathrm{CO}}$ and $M_{\star}$ are correct, another interesting limit arises: for the galaxy not to be dark matter dominated $\left(f_{\text {bary }}<50 \%\right)$ within its central few kpc, the system has to be seen at $i>35^{\circ}$ and the starburst disk has to be relatively compact $\left(r_{0}<2.3 \mathrm{kpc}\right)$. Assuming a probably more realistic $f_{\mathrm{DM}}=25 \% \pm 10 \%$ (e.g., Daddi et al. 2010) would suggest limiting $i \gtrsim 44^{\circ} \pm 4^{\circ}$ (at $r_{\text {Edd }}$ ) and $r_{0} \lesssim$ $1.5 \pm 0.2 \mathrm{kpc}\left(\right.$ at $\left.i=90^{\circ}\right)$.

\footnotetext{
11 Assuming that the system is seen close to edge-on.
}

Overall, the observations thus may favor a relatively compact, highly inclined galaxy with a high, perhaps dominant, fraction of molecular gas $\left(f_{\text {gas }}=0.3-0.8\right)$ that also dominates the baryonic mass in this system $\left(f_{\text {gas }} \simeq 0.84 f_{\text {bary }}\right)$. Despite the limited spatial resolution of this detection experiment, the diagnostic plot introduced here allows us to constrain the dynamical mass in this system to within a factor of $\sim 2-3$. However, dynamically resolved $\mathrm{CO}$ observations at high $\left(<0^{\prime \prime} .7\right)$ spatial resolution are necessary to determine to what degree the assumptions made here are correct. Also, potential effects of non-virial dynamics (such as in a merger) require further investigation.

\section{CONCLUSIONS}

We have detected a molecular gas reservoir in the highestredshift SMG AzTEC-3 $(z=5.298)$ that is comparable in mass to "typical" $z>2$ SMGs (e.g., Greve et al. 2005; Ivison et al. 2010; Harris et al. 2010) and likely has a low-excitation component similar ${ }^{12}$ in gas properties to that seen in another $z>4$ SMG (Carilli et al. 2010), but in addition has a highexcitation gas component with properties similar to $z>4$ quasar host galaxies (e.g., Riechers et al. 2006, 2009). The peak of the gas distribution is coincident with the rest-frame optical and far-infrared emission (stellar light and dust-enshrouded star formation), but slightly offset from the peak of the rest-frame UV emission (unobscured star formation), which appears to consist of multiple bright clumps. Given the high gas mass fraction and SFR of this galaxy, this suggests the presence of a heavily obscured starburst, possibly triggered by a major, "wet" (i.e., very gas rich), $\lesssim 8 \mathrm{kpc}$-scale merger. This is reminiscent of what is seen in "typical" $z>2$ SMGs (e.g., Tacconi et al. 2008).

The detection of luminous $\mathrm{CO}$ emission implies relatively advanced enrichment with heavy elements in the material that fuels the observed early burst of star formation in AzTEC-3. Assuming a Galactic abundance of $\mathrm{CO}$ yields $M(\mathrm{CO}) \simeq 7 \times$ $10^{7} M_{\odot}$. Such a level of early metal enrichment could be achieved through a few $\times 10^{7}$ hundred solar mass populationIII stars (e.g., Walter et al. 2003). This order-of-magnitude estimate, however, corresponds to $\gtrsim 10 \% M_{\star}$ and would require a quite "top-heavy" IMF. We thus speculate that asymptotic giant branch stars and supernovae may be extremely efficient in enriching their environments at such early epochs or that the stellar mass may be underpredicted due to obscuration in the most intensely star-forming regions.

The unusually high-excitation gas component in AzTEC-3 (for an SMG) raises the question whether or not the environment may play a role in determining its star formation properties. AzTEC-3 has two close, massive companions with consistent photometric redshifts within only $12.2 \mathrm{kpc}$. The molecular gas reservoir is clearly spatially separated from the companions; however, gravitational interactions may still play a role at such small distances, which may explain an above average peak gas density (leading to high $\mathrm{CO}$ excitation). The most similar object probably is the $z=4.055$ SMG GN20, which evolves in a comparably overdense environment (Daddi et al. 2009). Interestingly, the $\mathrm{CO}$ reservoir in AzTEC-3 is more compact, (partially) higher excited, and less than half as massive as that in GN20. This may either indicate that the (overdense) cosmic environments of both galaxies are considerably different (GN20 even has two massive SMG companions within $180 \mathrm{kpc}$

\footnotetext{
12 Future $\mathrm{CO}(J=1 \rightarrow 0)$ observations are desirable to characterize this
} component in more detail. 
distance) or that they are in different stages of their evolution as an SMG. The likely presence of a substantial low-excitation gas component besides highly excited gas in both systems may point at a fundamental difference in physical properties between the star-forming environments in $z>4$ SMGs and $z>4$ FIR-luminous quasar host galaxies (e.g., Riechers et al. 2006), which may trace overall different evolutionary stages of galaxies with comparable, high gas masses and SFRs. This difference may arise due to a higher average concentration of the gas in the quasar hosts (rather than large-scale influence of the active nucleus), e.g., due to later merger stages. This would be consistent with a scenario where SMGs evolve into FIRluminous quasars (e.g., Sanders et al. 1988; Coppin et al. 2008). Both populations are likely progenitors of the most massive galaxies in the present-day universe.

This investigation clearly motivates more detailed studies of the first SMGs and quasars, which will enable us to directly probe the scales that are critical to unravel the physical processes that drive the clustered evolution of massive galaxies in the early universe, back to the first billion years after the big bang.

We thank Josh Younger for the SMA image of AzTEC-3, Christian Henkel for the original version of the LVG code, and the referee for a helpful report. D.R. acknowledges support from NASA through Hubble Fellowship grant HST-HF-51235.01 awarded by STScI, operated by AURA for NASA, under contract NAS 5-26555. The IRAM PdBI is supported by INSU/ CNRS (France), MPG (Germany), and IGN (Spain). The EVLA is a facility of NRAO, operated by AUI, under a cooperative agreement with the NSF.

\section{REFERENCES}

Alexander, D. M., et al. 2008, AJ, 135, 1968

Blain, A. W., et al. 2002, Phys. Rep., 369, 111

Capak, P. L., et al. 2010, Nature, submitted

Carilli, C. L., et al. 2010, ApJ, 714, 1407

Chabrier, G. 2003, PASP, 115, 763

Coppin, K., et al. 2008, MNRAS, 389, 45

Daddi, E., et al. 2009, ApJ, 694, 1517

Daddi, E., et al. 2010, ApJ, 713, 686

Dannerbauer, H., et al. 2009, ApJ, 698, L178

Downes, D., \& Solomon, P. M. 1998, ApJ, 507, 615

Flower, D. R. 2001, J. Phys. B: At. Mol. Opt. Phys., 34, 2731

Greve, T. R., et al. 2005, MNRAS, 359, 1165

Harris, A. I., et al. 2010, ApJ, submitted (arXiv:1006.3691)

Ivison, R. J., et al. 2010, MNRAS, 404, 198

Michalowski, M. J., et al. 2010, ApJ, 712, 942

Riechers, D. A., et al. 2006, ApJ, 650, 604

Riechers, D. A., et al. 2008, ApJ, 686, L9

Riechers, D. A., et al. 2009, ApJ, 703, 1338

Sanders, D. B., et al. 1988, ApJ, 325, 74

Sanders, D. B., et al. 2007, ApJS, 172, 86

Schinnerer, E., et al. 2008, ApJ, 689, L5

Scott, K. S., et al. 2008, MNRAS, 385, 2225

Scoville, N. 2003, J. Korean Astron. Soc., 36, 167

Scoville, N., et al. 2007, ApJS, 172, 38

Solomon, P. M., \& Vanden Bout, P. A. 2005, ARA\&A, 43, 677

Spergel, D. N., et al. 2003, ApJS, 148, 175

Spergel, D. N., et al. 2007, ApJS, 170, 377

Tacconi, L. J., et al. 2006, ApJ, 640, 228

Tacconi, L. J., et al. 2008, ApJ, 680, 246

Thompson, T. A., Quataert, E., \& Murray, N. 2005, ApJ, 630, 167

Walter, F., et al. 2003, Nature, 424, 406

Walter, F., et al. 2009, Nature, 457, 699

Weiß, A., Walter, F., \& Scoville, N. Z. 2005, A\&A, 438, 533

Weiß, A., et al. 2007, A\&A, 467, 955

Younger, J. D., et al. 2007, ApJ, 671, 1531 